\title{
SEARCHES FOR SUPERSYMMETRY AT THE LHC
}

\author{
D.R. TOVEY * \\ Department of Physics and Astronomy, University of Sheffield, Hounsfield Road, \\ Sheffield $S 3$ YRH, UK.
}

\begin{abstract}
The considerable centre-of-mass energy and luminosity provided by the Large Hadron Collider will ensure a discovery reach for new particles which extends well into the multi- $\mathrm{TeV}$ region. ATLAS and CMS have carried out many studies of the implications of this capability for Beyond the Standard Model physics, focussing in particular on supersymmetric models. In this paper we summarise some key results of these studies.
\end{abstract}

\section{Introduction}

The ATLAS ${ }^{1}$ and CMS ${ }^{2}$ experiments at the Large Hadron Collider will give access to an unprecedented range of processes at $p_{T}$ scales and event rates far in excess of those generated by earlier colliders. Many rare Standard Model (SM) processes will be studied with high statistics while at the same time searches will be carried out for particles predicted to exist by theories extending the SM. The need to stabilise the mechanism for breaking electroweak $(\mathrm{EW})$ symmetry in the SM requires that at least some of these new particles have masses of order the EW scale $(\sim 100 \mathrm{GeV})$. Hence there is every reason to believe that they should be observable at the LHC.

In this paper we review the results of some of the studies of 'Beyond the Standard Model' physics at the LHC which have been carried out by ATLAS and CMS, concentrating on those involving supersymmetry (SUSY).

\section{Theoretical Motivation}

Supersymmetry is one of the oldest and best motivated of the theories predicting the existence of physics beyond the Standard Model ${ }^{3}$. SUSY models typically require the existence of at least one SUSY partner for each SM fermion and vector boson, together with a considerably enlarged

*On behalf of the ATLAS and CMS collaborations 


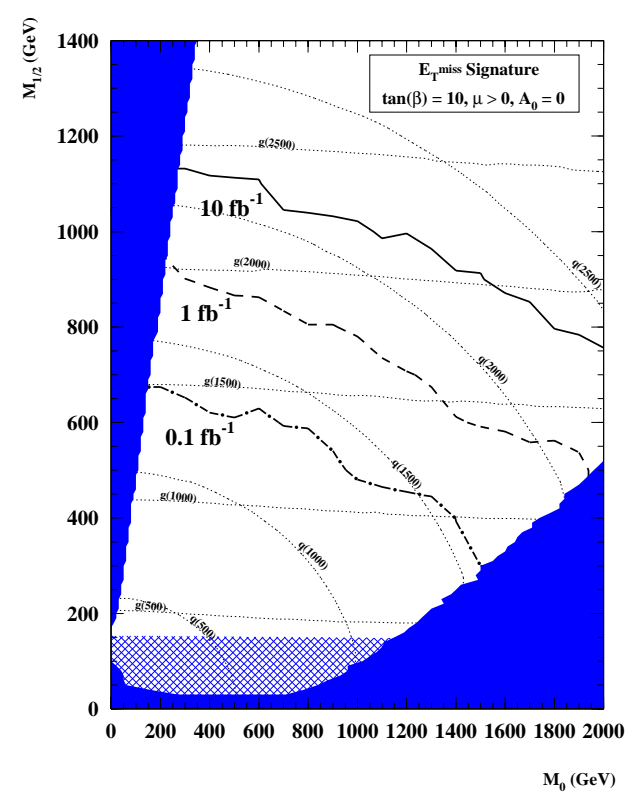

Figure 1. ATLAS $5 \sigma$ discovery potential of the inclusive jets $+E_{T}^{\text {miss }}$ channel in the $m_{0}-m_{1 / 2}$ plane for mSUGRA models with $\tan (\beta)=10, \mu>0$ and $A_{0}=0$ assuming $0.1 \mathrm{fb}^{-1}, 1 \mathrm{fb}^{-1}$ and $10 \mathrm{fb}^{-1}$ integrated luminosity. Full dark regions are excluded by theory, hatched regions by experiment (LEP2 and elsewhere).

Higgs sector. With the exception of spin these SUSY particles ('sparticles') possess the same quantum numbers as their SM counterparts. The fact that we have not observed such states in nature implies that SUSY is broken by a mechanism which causes sparticles to acquire masses greater than SM states. The precise nature of this mechanism is curently unknown but candidates include gravity-mediation (e.g. minimal Supergravity or mSUGRA models ${ }^{4}$ ) as well as gauge mediation (GMSB models ${ }^{5}$ ) and anomaly mediation (AMSB models ${ }^{6}$ ). A further feature of many SUSY models is the absolute conservation of a multiplicative quantum number known as R-parity at each SUSY vertex, which causes SUSY states to be pair produced and forces the Lightest Supersymmetric Particle (LSP) to be neutral and stable. Missing transverse energy generated by the escape of two such LSPs from each SUSY event provides the classic signature for R-parity conserving SUSY at hadron colliders. 


\section{Inclusive Signatures}

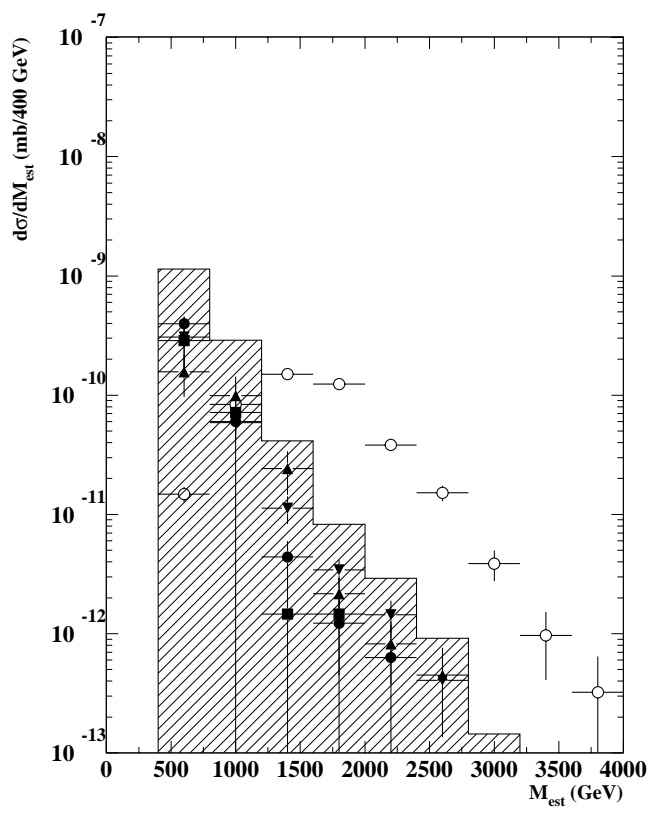

Figure 2. Effective mass distributions for SM background channels and SUSY signal at ATLAS mSUGRA point $5\left(m_{0}=100 \mathrm{GeV}, m_{1 / 2}=300 \mathrm{GeV}, A_{0}=300 \mathrm{GeV}, \mu>\right.$ $O$ and $\tan (\beta)=2.1)$. SM background contributions are $t \bar{t}$ (filled circles), $W+j e t$ (triangles), $Z+$ jet (downward triangles) and $Q C D$ (filled squares). The hatched histogram corresponds to the sum of all SM backgrounds while the open circles correspond to the SUSY signal population. Error bars indicate statistical errors arising from the finite Monte Carlo sample.

Inclusive searches for R-parity conserving SUSY using the generic $E_{T}^{\text {miss }}$ signature will be carried out by ATLAS and CMS from the outset of LHC operation. In addition to cuts on $E_{T}^{\text {miss }}$, cuts can be imposed on the multiplicity and $p_{T}$ of jets and leptons in order to reduce SM background from $\mathrm{W}+$ jet, $\mathrm{Z}+$ jet and $\mathrm{t} \overline{\mathrm{t}}$ processes and mismeasured QCD events. Studies have shown that within the mSUGRA framework both ATLAS and CMS should be sensitive to SUSY models with gluino and squark masses $\sim 2 \mathrm{TeV}$ after one year of low luminosity running $\left(10 \mathrm{fb}^{-1}\right.$ integrated luminosity - Fig. 1, taken from Ref. ${ }^{9}$ ), rising to $\sim 2.5 \mathrm{TeV}$ after one year of high luminosity 
running $\left(100 \mathrm{fb}^{-1}\right)^{7,8,9,10,11}$. Following discovery of a SUSY signal using this signature, the mass scale of the produced sparticles can then be measured in a model-independent fashion with an ultimate error $\lesssim 10 \%$ (mSUGRA models) by fitting to the distribution (Fig. 2, taken from Ref. ${ }^{9}$ ) of a variable based upon the scalar sum of jet $p_{T}$ and $E_{T}^{\text {miss }}$ in jet $+E_{T}^{\text {miss }}$ +0 lepton events ${ }^{9,11,12,13}$. The normalisation of this distribution also provides a measure of the total SUSY production cross-section and together these two pieces of information can be used to constrain the SUSY breaking machanism 9,13 .

\section{Sparticle Mass Measurements}

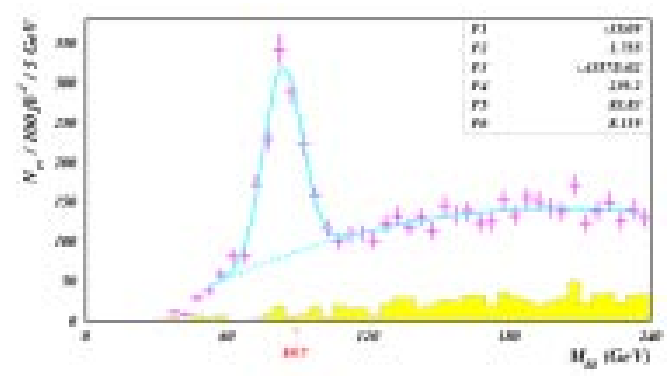

Figure 3. $b \bar{b}$ invariant mass spectrum for $m S U G R A$ point with $m_{0}=m_{1 / 2}=500 \mathrm{GeV}$, $\tan (\beta)=2, \mu<0$ and $A_{0}=0$. The prominent peak arises from $\tilde{\chi}_{2}^{0} \rightarrow \tilde{\chi}_{1}^{0} h$ decays. The shaded histogram shows the contribution from SM background processes.

Further information regarding the SUSY model and its parameters can be obtained by measuring SUSY particle masses. In R-parity conserving models the presence of two invisible LSPs prevents the reconstruction of invariant mass peaks, however combinations of sparticle masses can be measured by searching for edges in dilepton invariant mass spectra ${ }^{7,12}$. In mSUGRA models the most prominent such edge arises from $\tilde{\chi}_{2}^{0}$ decays, with its position depending on the masses of the $\tilde{\chi}_{2}^{0}$ and its SUSY decay products, such as the $\tilde{\chi}_{1}^{0}$ and light sleptons. By selecting events in the region of the dilepton edge and forming invariant mass combinations of (di)leptons with jets it is also possible to gain additional sensitivity to the masses of left-handed squarks. Additional observation of thresholds in such invariant mass spectra then provides sufficient constraints for the full sparticle mass spectrum to be reconstructed ${ }^{14}$. Similar procedures have been developed 
for mass reconstruction when $\tilde{\chi}_{2}^{0}$ decays to the light higgs are kinematically accessible $^{7,11}$ (Fig. 3, taken from Ref. ${ }^{11}$ ).

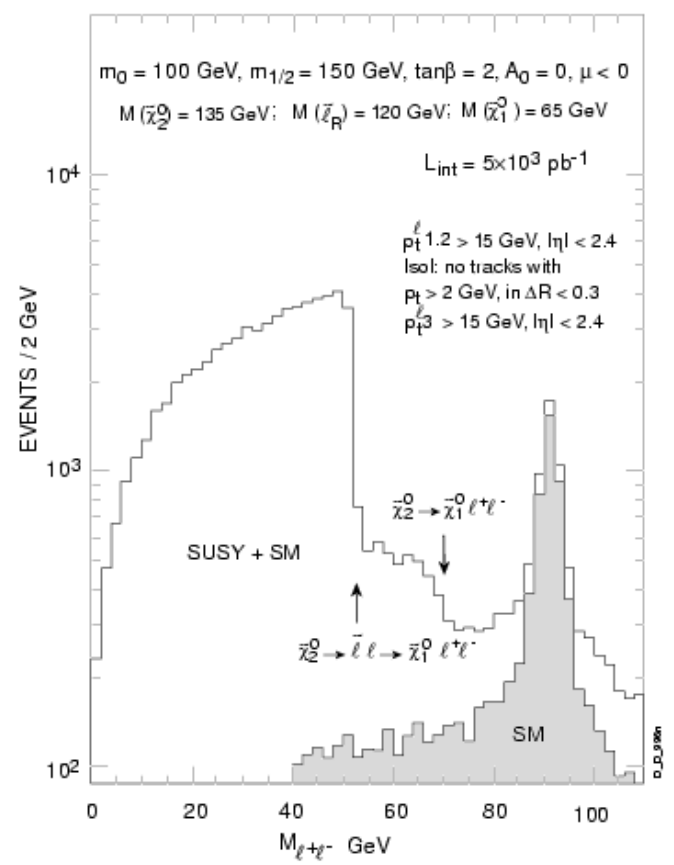

Figure 4. Dilepton invariant mass spectrum of events generated with an mSUGRA model with parameters shown. The left and right hand edges arise from two and three body decays of $\tilde{\chi}_{2}^{0}$ respectively.

Work by CMS has investigated the origin and observability of dilepton edges over the mSUGRA $m_{0}-m_{1 / 2}$ plane ${ }^{15}$. When kinematically allowed the $\tilde{\chi}_{2}^{0}$ decay proceeds via two sequential two-body processes involving slepton exchange:

$$
\tilde{\chi}_{2}^{0} \rightarrow \tilde{l}^{+(-)} l^{-(+)} \rightarrow \tilde{\chi}_{1}^{0} l^{+} l^{-}
$$

while elsewhere a direct three body decay to the $\tilde{\chi}_{1}^{0}$ is favoured:

$$
\tilde{\chi}_{2}^{0} \rightarrow \tilde{\chi}_{1}^{0} l^{+} l^{-}
$$

These two cases can be distinguished experimentally by measuring the $p_{T}$ asymmetry between the two leptons ${ }^{11}$. In certain very limited regions of parameter space the $\sigma$.BR of both decay channels can be appreciable 
and in this case two edges are observed, giving additional constraints on the sparticle mass spectrum (Fig. 4, taken from Ref. ${ }^{11}$ ). In addition, measurements of the cross-section for events in the most prominent edge can be compared with the height of the $\mathrm{Z}$ peak arising from higher gaugino decays in order to constrain the value of $\tan (\beta)^{15}$.

\section{Acknowledgements}

The author wishes to thank Frank Paige and Giacomo Polesello for discussions. He also wishes to thank members of the ATLAS and CMS collaborations for the work described in this paper.

\section{References}

1. ATLAS Collaboration, Technical Proposal, CERN/LHCC/94-43.

2. CMS Collaboration, Technical Proposal, CERN/LHCC/94-38.

3. H.P. Nilles, Phys. Rep. 111 (1984) 1; H.E. Haber and G.L. Kane, Phys. Rep. 117 (1985) 75.

4. see e.g. L. Alvarez-Gaume et al., Nucl. Phys. B221 (1983) 495.

5. M. Dine, W. Fischler and M. Srednicki, Nucl. Phys. B189 (1981) 575; S. Dimopoulos and S. Raby, Nucl. Phys. B192 (1981) 353.

6. L. Randall and R. Sundrum, Nucl. Phys. B557 (1999) 79; G.F. Giudice, M.A. Luty, H. Murayama and R. Rattazzi, JHEP 12 (1998) 027.

7. ATLAS Collaboration, Detector and Physics Performance Technical Design Report, CERN/LHCC/99-15.

8. H. Baer et al., Phys. Rev. D52 (1995) 2746; ibid. Phys. Rev. D53 (1996) 6241.

9. D.R. Tovey, ATLAS SCIENTIFIC NOTE SN-ATLAS-2002-020.

10. S. Abdullin and F. Charles, Nucl. Phys. B547 (1999) 60; M. Dzelalija et al., Mod. Phys. Lett. A15 (2000) 465.

11. S. Abdullin et al., J. Phys. G28 (2002) 469.

12. I. Hinchliffe et al., Phys. Rev. D55 (1997) 55209.

13. D.R. Tovey, Phys. Lett. B498 (2001) 1.

14. C.G. Lester et al, JHEP 0009 (2000) 004.

15. D. Denegri, et al., Phys. Rev. D60 (1999) 035008. 